\title{
Bio-écologie des diatomées épilithiques de la rivière Mfoundi (Yaoundé, Cameroun) : diversité, distribution spatiale et influence des pollutions organiques \\ Bio-ecology of epilithic diatoms in the Mfoundi River (Yaoundé, Cameroon): diversity, spatial distribution and influence of organic pollution
}

\author{
Daniel Ébang Menye, Serge Hubert Zébazé Togouet, Samuel Foto Menbohan, \\ Norbert Kemka, Moïse Nola, Claude Boutin, Victor François Nguetsop, Moussa \\ Djaouda et Thomas Njiné
}

Volume 25, numéro 3, 2012

Reçu le 17 décembre 2011, accepté le 14 septembre 2012

URI : https://id.erudit.org/iderudit/1013103ar

DOI : https://doi.org/10.7202/1013103ar

Aller au sommaire du numéro

Éditeur(s)

Université du Québec - INRS-Eau, Terre et Environnement (INRS-ETE)

ISSN

1718-8598 (numérique)

Découvrir la revue

Citer cet article

Menye, D. É., Zébazé Togouet, S. H., Menbohan, S. F., Kemka, N., Nola, M., Boutin, C., Nguetsop, V. F., Djaouda, M. \& Njiné, T. (2012). Bio-écologie des diatomées épilithiques de la rivière Mfoundi (Yaoundé, Cameroun) : diversité, distribution spatiale et influence des pollutions organiques. Revue des sciences de l'eau / Journal of Water Science, 25(3), 203-218.

https://doi.org/10.7202/1013103ar

\section{Résumé de l'article}

Sont considérés dans cette étude, l'impact des rejets polluants d'origines urbaine et industrielle sur la qualité des eaux du Mfoundi, l'abondance, la richesse spécifique et la structure des communautés diatomiques qui peuplent ce cours d'eau. Le calcul du pourcentage de taxons tolérants à la pollution organique a permis d'évaluer la réponse de ces algues à l'enrichissement du milieu en matières organiques. Les analyses physico-chimiques des eaux mettent en évidence l'existence d'un gradient de pollution croissant de l'amont à l'aval de la rivière. Le cours supérieur présente des eaux turbides avec des teneurs élevées de matières en suspension. Les cours moyen et inférieur sont fortement contaminés par les matières organiques qu'apportent les eaux usées domestiques et les effluents industriels qu'ils reçoivent. Au total, 237 taxons de diatomées épilithiques, majoritairement cosmopolites, appartenant à 39 genres répartis dans 25 familles, ont été inventoriés. Le développement dans le cours supérieur du Mfoundi de taxons tels que Gomphonema parvulum (Kützing) Kützing et variétés, Gomphonema gracile Ehrenberg et Navicula radiosa Kützing, est associé essentiellement à la turbidité des eaux de ce tronçon. La distribution de Nitzschia palea (Kützing) W. Smith, Navicula geoppertiana (Bleisch) Grunow, Navicula mutica Kützing et Pinnularia subcapitata Gregory dans les cours moyen et inférieur est étroitement liée à la charge organique et la forte minéralisation des eaux. $N$. palea est l'espèce la plus abondante dans les stations Mf5 (66 \%) et Mf6 (53\%), indiquant une importante pollution organique de ces sites sous l'effet des rejets des industries brassicoles et vinicoles opérant dans leur voisinage. La variation spatiale de la diversité spécifique n'a pas souligné toute l'ampleur de l'altération de la qualité des eaux; toutefois, l'analyse du pourcentage de taxons polluo-tolérants révèle que les apports graduels en nutriments et en matières organiques dans les cours moyen et inférieur contribuent à l'eutrophisation progressive de ces secteurs. Il est montré que les diatomées épilithiques sont sensibles à la variation de la qualité de l'eau du Mfoundi, et peuvent être utilisées comme bioindicateurs de l'état de santé des cours d'eau urbains du Cameroun. 


\title{
BIO-ÉCOLOGIE DES DIATOMÉES ÉPILITHIQUES DE LA RIVIĖRE MFOUNDI (YAOUNDÉ, CAMEROUN) : DIVERSITÉ, DISTRIBUTION SPATIALE ET INFLUENCE DES POLLUTIONS ORGANIQUES
}

\author{
Bio-ecology of epilithic diatoms in the Mfoundi River (Yaoundé, Cameroon): diversity, spatial distribution and influence of organic pollution
}

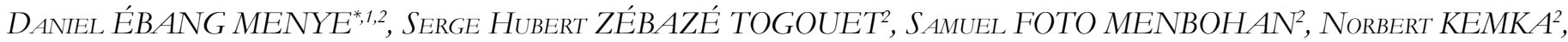
MoÏsE NOL $A^{2}$, CLAUDE BOUTIN ${ }^{4,5}$, VICTOR Fr ANCOIS NGUETSOP 3 , MOUSSA DJAOUDA $A^{1,2}$ ET THOMAS NJINÉ ${ }^{2}$

${ }^{1}$ Département des Sciences de la Vie et de la Terre, École Normale Supérieure, Université de Maroua, B.P. 55, Maroua, Cameroun.

${ }^{2}$ Unité d'Hydrobiologie et Qualité des Eaux, Laboratoire de Biologie Générale, Faculté des Sciences, Université de Yaoundé I, B.P. 812, Yaoundé, Cameroun.

${ }^{3}$ Laboratoire de Botanique Appliquée, Faculté des Sciences, Université de Dschang, B.P. 67, Dschang, Cameroun. ${ }^{4}$ Laboratoire d'Écologie Fonctionnelle et Environnement, ECOLAB (UMR CNRS-UPS-INPT-INSA n 5245), Université Paul Sabatier, Toulouse III, Bât. 4R1, 118 Route de Narbonne, 31062, Toulouse Cedex 04, France.

${ }^{5} 48$ rue Mouffetard, 75005 Paris, France.

Reçu le 17 décembre 2011, accepté le 14 septembre 2012

\section{RÉSUMÉ}

Sont considérés dans cette étude, l'impact des rejets polluants d'origines urbaine et industrielle sur la qualité des eaux du Mfoundi, l'abondance, la richesse spécifique et la structure des communautés diatomiques qui peuplent ce cours d'eau. Le calcul du pourcentage de taxons tolérants à la pollution organique a permis d'évaluer la réponse de ces algues à l'enrichissement du milieu en matières organiques. Les analyses physico-chimiques des eaux mettent en évidence l'existence d'un gradient de pollution croissant de l'amont à l'aval de la rivière. Le cours supérieur présente des eaux turbides avec des teneurs élevées de matières en suspension. Les cours moyen et inférieur sont fortement contaminés par les matières organiques qu'apportent les eaux usées domestiques et les effluents industriels qu'ils reçoivent. Au total, 237 taxons de diatomées épilithiques, majoritairement cosmopolites, appartenant à 39 genres répartis dans 25 familles, ont été inventoriés. Le développement dans le cours supérieur du Mfoundi de taxons tels que Gomphonema parvulum (Kützing) Kützing et variétés, Gomphonema gracile Ehrenberg et Navicula radiosa Kützing, est associé essentiellement à la turbidité des eaux de ce tronçon. La distribution de Nitzschia palea (Kützing) W. Smith, Navicula geoppertiana (Bleisch) Grunow, Navicula mutica Kützing et Pinnularia subcapitata Gregory dans les cours moyen et inférieur est étroitement liée à la charge organique et la forte minéralisation des eaux. $N$. palea est l'espèce la plus abondante dans les stations Mf5 (66 \%) et Mf6 (53\%), indiquant une importante pollution organique de ces sites sous l'effet des rejets des industries brassicoles et vinicoles opérant dans leur voisinage. La variation spatiale de la diversité spécifique n'a pas souligné toute l'ampleur de l'altération de la qualité des eaux; toutefois, l'analyse du pourcentage de taxons polluo-tolérants révèle que les apports 
graduels en nutriments et en matières organiques dans les cours moyen et inférieur contribuent à l'eutrophisation progressive de ces secteurs. Il est montré que les diatomées épilithiques sont sensibles à la variation de la qualité de l'eau du Mfoundi, et peuvent être utilisées comme bioindicateurs de l'état de santé des cours d'eau urbains du Cameroun.

\section{Mots-clés : diatomées épilithiques, diversité spécifique, qualité de l'eau, rivière Mfoundi, limnologie équatoriale, Cameroun.}

\section{ABSTRACT}

The Mfoundi River is used for the disposal of solid wastes and waste waters from domestic and industrial activities, which can alter its water quality and biodiversity. The aim of this study was to assess the structure of diatom communities in the Mfoundi River in relation to some physico-chemical variables, i.e., analyzing the changes in species composition and the dominance profile of the main species along a pollution gradient. The diversity metrics (species richness, Shannon's diversity index, evenness) and the percent pollution tolerant valves (\%PTV) were also tested to characterize the water quality and the response of diatoms to environmental enrichment in organic matter. The physico-chemical analyses of the waters revealed a gradient of increasing pollution from upstream to downstream of the river. The upper reach presented turbid waters with high levels of suspended solids. The middle and the lower reaches were highly contaminated with organic matters originating from domestic sewage and industrial effluents. A total of 237 epilithic diatom taxa, mostly cosmopolitan, belonging to 39 genera distributed among 25 families were inventoried. The development in the upper reach of the Mfoundi of taxa such as Gomphonema parvulum (Kützing) Kützing and varieties, Gomphonema gracile Ehrenberg, and Navicula radiosa Kützing reflected in particular the turbid waters of this section. The distribution of Nitzschia palea (Kützing) W. Smith, Navicula geoppertiana (Bleisch) Grunow, Navicula mutica Kützing, and Pinnularia subcapitata Gregory in the middle and lower reaches was closely related to the organic load and high degree of mineralisation of the waters. $N$. palea was the most abundant species at the stations Mf5 (66\%) and Mf6 (53\%), indicating a significant organic contamination of these sites under the effect of discharges from brewery and wine-making industries operating within their vicinity. The spatial variation of diversity metrics did not reflect the full extent of the alteration of water quality, but the analysis of the $\%$ PTV revealed that incremental inputs of nutrients and organic matter in the middle and lower reaches of the Mfoundi may be contributing to the start of the eutrophication process in these sectors. This study showed that epilithic diatoms are sensitive to the variations in water quality of the Mfoundi, and can be used as bioindicators of the health status of urban streams in Cameroon.

\section{Key words: epilithic diatoms, specific diversity, water quality, Mfoundi River, equatorial limnology, Cameroon.}

\section{INTRODUCTION}

L'état de santé des cours d'eau en zone urbaine est en permanence susceptible d'être influencé par des rejets polluants non traités (eaux usées domestiques et effluents industriels notamment) qui dégradent la qualité des eaux et modifient la biodiversité de ces écosystèmes (BEYENE et al., 2009; WINTER et DUTHIE, 1998). Au Cameroun comme dans la plupart des pays en voie de développement, les cours d'eau servent très souvent de dépotoir de déchets liquides et solides, particulièrement en milieu urbain (AGENDIA et al., 2000; FOTO MENBOHAN, 1997). Les rejets solides augmentent la turbidité des eaux et la teneur des matières en suspension (pollution mécanique), alors que les déchets liquides entraînent généralement des pollutions organiques et métalliques au niveau du milieu récepteur (LE PIMPEC et al., 2002). Cette situation est aggravée d'année en année par la poussée démographique, la multiplication des activités et des structures polluantes liées à la croissance des villes, l'insuffisance des stations d'épuration fonctionnelles, l'inexistence d'un système d'assainissement approprié et l'augmentation des surfaces imperméables des bassins versants (TANAWA et al., 2003; TCHOTSOUA, 1993).

Pour mieux surveiller les cours d'eau urbains de façon à répondre aux exigences de la législation camerounaise sur la protection des eaux de surface contre les pollutions (DECRET 165/PM, 2001), il est nécessaire de déterminer au préalable " l'état symptomatique " des eaux de ces hydrosystèmes, et d'identifier et quantifier les organismes qui y vivent (CAMARGO, 1994; NDIRITU et al., 2003). Les analyses physico-chimiques des eaux permettent de connaître la nature et la concentration des contaminants présents dans l'eau au moment où les prélèvements ont été effectués. Par l'étude des organismes aquatiques, on procède à une meilleure évaluation de l'intégrité des écosystèmes car leur présence ou leur abondance reflète les conditions du milieu qui se sont succédées durant leur développement (BEYENE et al., 2009; JÜTTNER et al., 1996; SOININEN, 2002). Parmi les organismes utilisés comme "bioindicateurs " de la qualité des eaux, les algues, particulièrement les diatomées, occupent une place de choix pour diagnostiquer l'état de santé des rivières, car elles répondent de manière rapide à l'eutrophisation des eaux ainsi qu'aux diverses pollutions (MORIN, 2006; ROTT et al., 1998; ROUND, 1991). De plus, les diatomées constituent l'élément essentiel de la flore algale des rivières à la base des 
chaînes alimentaires en milieu aquatique (EULIN, 1997; HARDING et al., 2005). Elles ont une taille microscopique permettant un échantillonnage quantitatif simple et rapide, un cycle de reproduction court, et sont riches en espèces présentant des degrés différenciés de sensibilité à des gammes de paramètres de la qualité des eaux (BLINN et BAILEY, 2001; DE LA REY et al., 2004).

Peu d'études ont été faites sur la florule diatomique des lacs et cours d'eau du Cameroun. Par ailleurs, les données existantes sont anciennes, et ne se rapportent qu'au Lac Ossa (NGUÉTSOP, 1997), dans la région d’Edéa, et à la région du lac Tchad (COMPÈRE, 1975). L'étude présentée ici a permis d'évaluer l'impact des rejets polluants d'origine urbaine sur la structure des communautés de diatomées épilithiques, et la qualité des eaux du Mfoundi, un cours d'eau urbain fortement perturbé en zone équatoriale. Les objectifs spécifiques de ce travail étaient : (1) de déterminer la composition spécifique et l'abondance des diatomées dans les différents sites de prélèvements; (2) d'établir le profil de dominance des espèces et variétés de diatomées le long du gradient de pollution; (3) d'évaluer la corrélation pouvant exister entre les principales variables physico-chimiques de l'eau et l'abondance des espèces dominantes présentes dans l'ensemble des stations. Les données recueillies pourront servir à l'élaboration d'un outil de contrôle de ce cours d'eau qui traverse la ville de Yaoundé, grande métropole du Cameroun, et ses affluents.

\section{MATÉRIEL ET MÉTHODES}

\subsection{Site d'étude}

La région de Yaoundé (351’ N, 11³2’ E) est située sur la bordure ouest du plateau sud-camerounais, à $760 \mathrm{~m}$ d'altitude, et à $250 \mathrm{~km}$ de la côte atlantique (OLIVRY, 1986). Son socle est constitué essentiellement de formations métamorphiques et cristallines (VICAT et BILONG, 1998) qui donnent des sols acides avec un $\mathrm{pH}$ généralement inférieur à 6 unités conventionnelles (BACHELIER, 1959). Le climat est équatorial classique de type guinéen, chaud et humide, caractérisé par quatre saisons (deux saisons pluvieuses et deux saisons sèches) de durées variables d'une année à l'autre (SUCHEL, 1987).

Le Mfoundi est le principal cours d'eau de la ville de Yaoundé. Son bassin versant couvre une superficie de $97,7 \mathrm{~km}^{2}$ dont les $95 \%$ sont entièrement anthropisés; les espaces imperméabilisés (bitumage, habitations) occupent environ $60 \%$. Cet hydrosystème collecte les déchets solides et liquides non traités de son bassin versant, et les flux polluants drainés par ses affluents. Ces rejets sont constitués essentiellement par les eaux usées domestiques et les eaux résiduaires des industries alimentaires. Des tas d'ordures recouvrent en plusieurs endroits les berges et parfois même le lit de ce cours d'eau, diminuant la vitesse d'écoulement des eaux.

Suivant l'occupation de l'espace, il est aisé de distinguer dans le bassin du Mfoundi deux grandes zones : le cours supérieur, où se pratique encore une agriculture maraîchère artisanale utilisant parfois des pesticides et des engrais, et la partie aval qui se caractérise par une intense activité industrielle. Dans cette zone se trouvent notamment les usines de la Société Anonyme des Brasseries du Cameroun (SABC), la Société de Fabrication du Vin du Cameroun (SOFAVINC) et les entrepôts de la Société Camerounaise des Dépôts Pétroliers (SCDP). Sept stations d'échantillonnage ont été choisies le long du cours d'eau (Figure 1). La station 1 (Mf1), située à $1800 \mathrm{~m}$ de la source, dans une zone faiblement peuplée où les habitations sont de types provisoire et spontané, reçoit à la fois des eaux usées ménagères et de ruissellement de parcelles cultivées. La station 2 (Mf2) est localisée dans le cours moyen à $8 \mathrm{~km}$ de la source, en aval du site de décharge des effluents du marché de Nkol-Eton et de la gare ferroviaire de marchandises du transcamerounais. La station 3 (Mf3) se trouve juste à l'aval de la confluence avec l'Abiergué et le Mingoa, deux affluents de la rive droite fortement contaminés par des rejets domestiques et municipaux. Les stations Mf4 et Mf5 encadrent la SABC, la station Mf6 étant située en aval du point de décharge des effluents de la SCDP et de SOFAVINC. Avant d'atteindre la dernière station (Mf7), le Mfoundi traverse une zone marécageuse longue de plus de $3 \mathrm{~km}$, à végétation abondante riche en Echinocloa pyramidalis et Paspalum conjugatum, macrophytes souvent utilisés dans les stations expérimentales de phytoépuration des eaux usées à Yaoundé.

\subsection{Prélèvements d'eau et des diatomées épilithiques}

Les investigations ont été menées sur une période de neuf mois, d'avril à décembre 2006. Les prélèvements mensuels d'eau et de diatomées ont été effectués au niveau des stations susmentionnées. Les échantillons d'eau prélevés ont fait l'objet d'analyses physico-chimiques selon les méthodes de APHA-AWWA (1985) et de RODIER (1996) afin de mesurer la température, le $\mathrm{pH}$, la conductivité électrique, les solides totaux dissous, les matières en suspension, la turbidité, la couleur, l'oxygène et le gaz carbonique dissous, l'alcalinité, les duretés totale, magnésienne et calcique, l'azote ammoniacal, les orthophosphates, l'oxydabilité et la demande biochimique en oxygène pendant cinq jours $\left(\mathrm{DBO}_{5}\right)$. Les diatomées ont été prélevées en faciès lotique et en débit stabilisé, par grattage des parties supérieures des pierres ou des cailloux sur des surfaces d'environ $100 \mathrm{~cm}^{2}$. Le matériel ainsi récolté a été traité au péroxyde d'hydrogène $\left(\mathrm{H}_{2} \mathrm{O}_{2}, 130 \mathrm{~V}\right)$ bouillante. Ce traitement permet de digérer le protoplasme et le revêtement organique externe des cellules et d'obtenir leur squelette siliceux. Les échantillons de diatomées nettoyées 


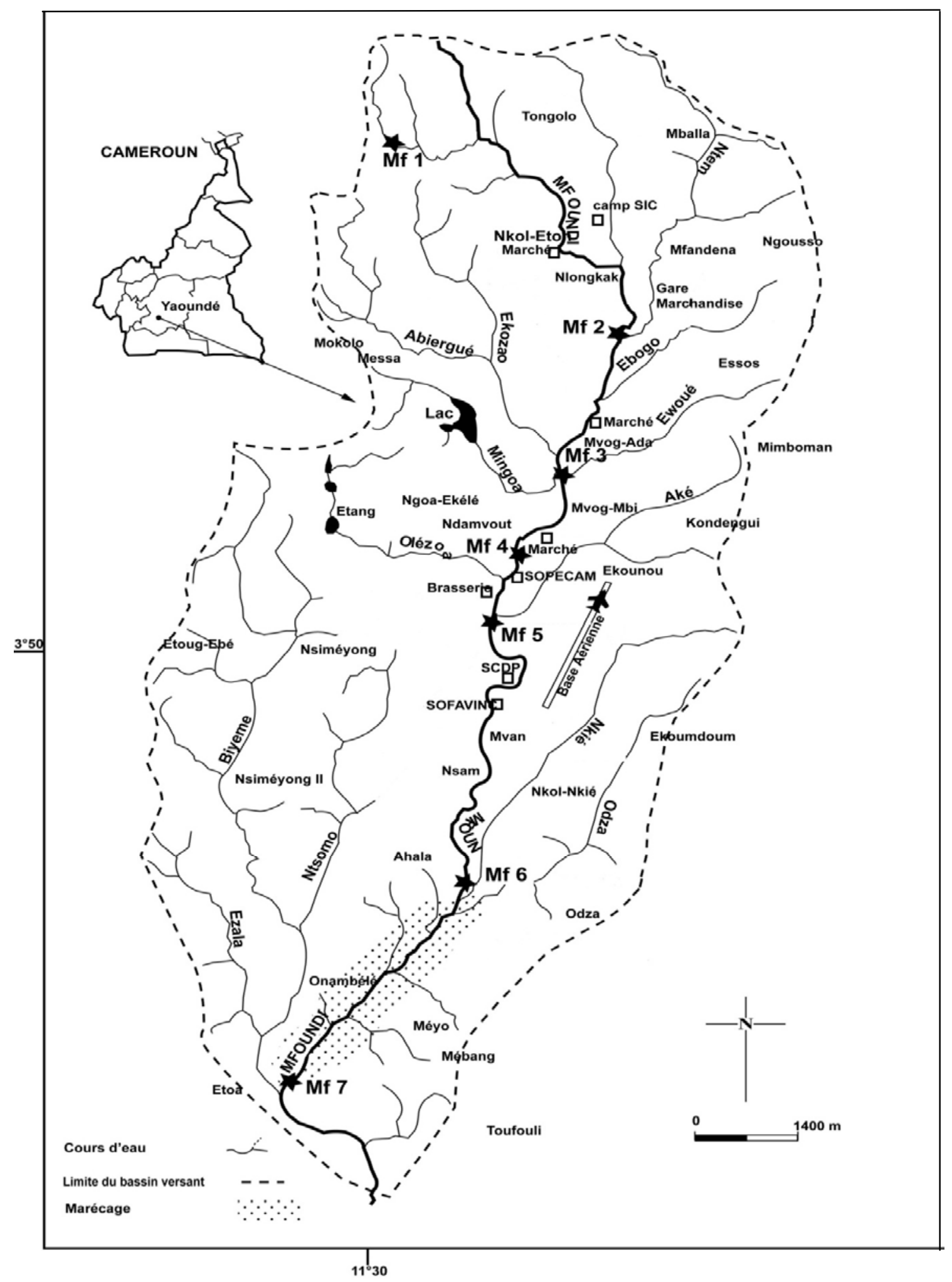

Figure 1. Bassin hydrographique du Mfoundi, à Yaoundé, et emplacements des sept stations de prélèvements (Mf1 à Mf7, stations Mfoundi1 à Mfoundi7).

Drainage basin of the Mfoundi River, in Yaoundé, and location of the sampling stations (Mf1 to Mf7, stations Mfoundil to Mfoundi7). 
ont ensuite été montés entre lames et lamelles en utilisant le Naphrax ${ }^{\bullet}$ comme medium (AFNOR, 2003). L'identification et le comptage des diatomées ( 400 frustules au minimum par échantillon) ont été effectués à l'aide d'un microscope optique Leitz Orthoplan, équipé d'un objectif à immersion de $100 \mathrm{X}$. La détermination des espèces et des sous-espèces a été réalisée suivant les critères morphologiques décrits par BOURRELLY (1968), COMPÈRE (1975), SCHOEMAN (1973), complétés par les descriptions de CARTER et DENNY (1982-1992), GASSE (1986), KRAMMER et LANGE-BERTALOT (19861991), et LANGE-BERTALOT et METZELTIN (1996).

\subsection{Analyse des données}

Les comptages ont été exprimés en abondance relative $\left(\mathrm{P}_{\mathrm{i}}=\mathrm{n}_{\mathrm{i}} / \mathrm{N}\right.$ où $\mathrm{n}_{\mathrm{i}}$ est l'effectif de l'espèce de rang $\mathrm{i}$, et $\mathrm{N}$ l'effectif total) de chaque espèce ou taxon. Pour évaluer la diversité des diatomées dans les stations prospectées, nous avons déterminé la richesse spécifique (RS), l'indice $\mathrm{H}^{\prime}$ de SHANNON et WEAVER (1949) et l'équitabilité $E\left(H ' / \log _{2}\right.$ de $S$, où $S$ est le nombre d'espèces ou taxons subspécifiques). L'indice $H^{\prime}$ et l'équitabilité E ont permis respectivement de rendre compte de l'importance numérique des taxons et d'estimer l'équirépartition des effectifs entre les taxons présents afin de comparer la diversité des peuplements diatomiques des différentes stations (DAJOZ, 2000). L'influence de la pollution organique sur la distribution des diatomées a été estimée à partir du pourcentage de Valves d'espèces Tolérantes à la Pollution (\%VTP). La sélection de ces espèces a été faite selon KELLY (1998) et VAN DAM (1994). Les prédicteurs susmentionnés ont permis de caractériser la structure de la communauté diatomique du Mfoundi. Les analyses en composantes principales (ACP) ont été effectuées à l'aide du logiciel XLstat-2007 avec les données de la physicochimie, d'une part, et de l'abondance relative des principales espèces et variétés de diatomées (ayant au minimum $2 \%$ d'abondance relative cumulée dans au moins un prélèvement), d'autre part. Ces ACP ont permis de s'assurer des effets des variables physico-chimiques dans un premier temps puis d'établir les différences taxonomiques entre les stations. Tous les échantillons d'eau (63) et de diatomées (63) ont été pris en compte. Le degré de liaison entre les variables physicochimiques et biologiques recueillies mensuellement a été évalué à l'aide du test de corrélation de rang (rs) de Spearman en utilisant le logiciel SPSS version 12.0.

\section{RÉSULTATS}

\subsection{Physico-chimie des eaux}

Les résultats des analyses physico-chimiques des eaux du Mfoundi sont réunis dans le tableau 1. La température relevée au niveau des stations d'étude prend une valeur moyenne qui varie de 21 à $25^{\circ} \mathrm{C}$, les valeurs les plus élevées étant enregistrées dans les stations Mf4 à Mf6. Le pH est proche de la neutralité. Les matières en suspension (MES), la turbidité et la couleur ont leurs plus fortes valeurs dans le cours supérieur, notamment à la station Mf1. Le long du cours d'eau, on observe une augmentation régulière des valeurs des variables indicatrices de la pollution organique que sont l'azote ammoniacal, la $\mathrm{DBO}_{5}$ et l'oxydabilité. Il en est de même de la conductivité électrique et des solides totaux dissous, dont les valeurs mettent en évidence une variation croissante de la minéralisation de l'eau, de l'amont vers l'aval de l'hydrosystème. À l'opposé, une diminution concomitante du taux de saturation en oxygène dissous est enregistrée. Dans l'ensemble, l'alcalinité, la dureté, les orthophosphates, la $\mathrm{DBO}_{5}$, l'oxydabilité et l'azote ammoniacal montrent des valeurs maximales dans les stations Mf2 à Mf6. Ces variables affichent de faibles valeurs à la station Mf7 après que le Mfoundi ait traversé une zone marécageuse. Ces résultats laissent apparaître l'existence d'un gradient de pollution le long du cours d'eau, du cours supérieur au cours inférieur.

L'ACP effectuée avec les 17 variables physico-chimiques mesurées a permis de caractériser la qualité del'eau des différentes stations et de classer tous les échantillons d'eau (Figure 2). Les axes $\mathrm{F} 1$ et $\mathrm{F} 2$ ont été retenus car ils représentent 35,49\% de la variabilité expliquée la plus élevée. L'axe F1 (20,49 \% d'inertie) est déterminé par les paramètres révélateurs de la pollution organique et de la minéralisation. Il indique la qualité de l'eau dans le cours inférieur du Mfoundi. L'axe F2 (15,00\% d'inertie) individualise les matières en suspension (MES), la couleur (Coul) et la turbidité (Turb). Cet axe caractérise les eaux turbides et colorées des stations Mf1 et Mf7 (Figure 2A). La répartition des échantillons d'eau de différentes stations sur le plan factoriel 1-2 de l'ACP (Figure 2B) révèle une variation spatio-temporelle des paramètres physico-chimiques de l'eau au niveau des différentes stations. En considérant la position de ces échantillons d'eau, on ne distingue pas clairement une variabilité saisonnière des conditions environnementales mais plutôt un changement de la qualité de l'eau le long du Mfoundi sous l'effet de diverses sources de polluants. Les échantillons d'eau ont été classés en trois groupes : Le groupe 1 rassemble les échantillons des stations Mf1 et Mf7 qui présentent des eaux turbides, colorées et faiblement minéralisées. Les fortes valeurs de la couleur, des MES et de la turbidité y sont enregistrées surtout pendant les saisons des pluies, au cours des mois d'avril, mai et juin, pour la petite saison des pluies, et en septembre et octobre, pour la grande saison des pluies. Les groupes 2 et 3 réunissent les échantillons des stations Mf2 à Mf6. Quel que soit le mois de prélèvement, ces stations ont des eaux à forte conductivité électrique, des teneurs élevées en ions alcalino-terreux, et des paramètres révélateurs d'une pollution organique. Ces eaux s'appauvrissent également en oxygène dissous, ce qui semble être une conséquence de la dégradation aérobie des substances organiques contenues dans les rejets. Ces 


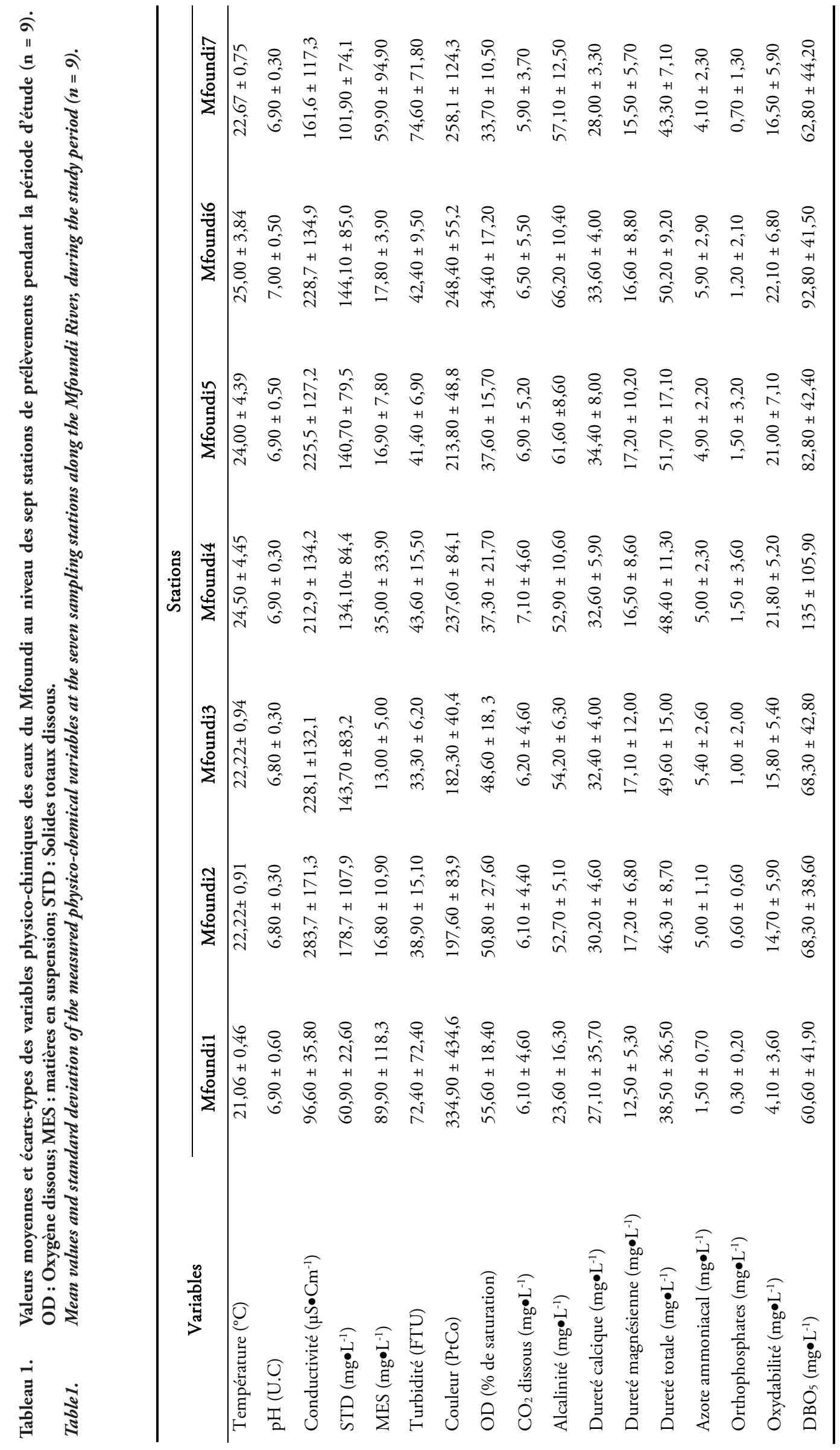



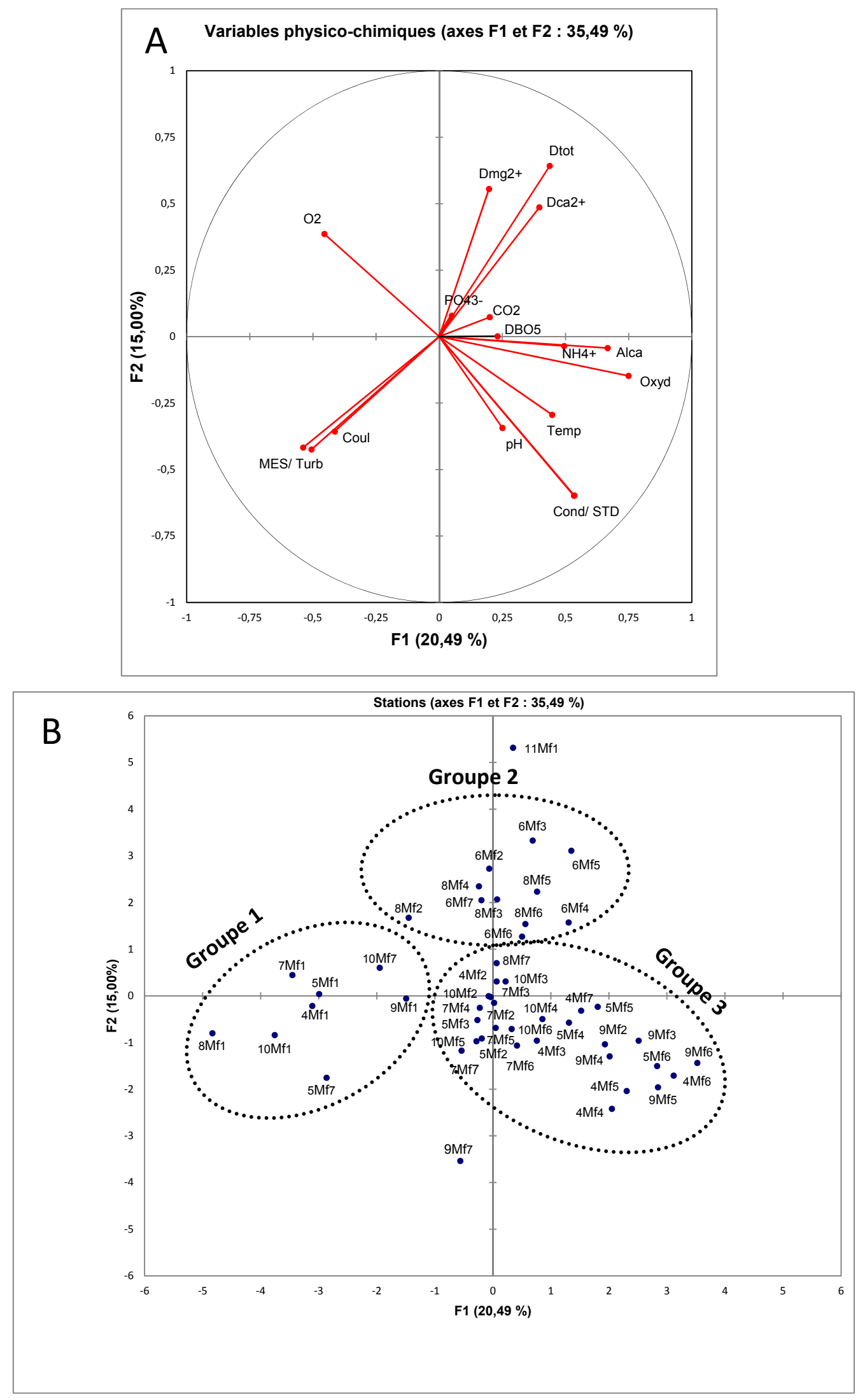

Figure 2. A- Projections des variables physico-chimiques des échantillons d'eau collectés sur le plan de deux premiers axes factoriels de l'ACP (axe 1 horizontal et axe 2 vertical). B- Projections des trois groupes de stations, décrites par leurs caractéristiques physico-chimiques, sur le plan de deux premiers axes factoriels de l'ACP (axe 1 horizontal et axe 2 vertical). Les nombres 4 à 9 précédant la station représentent le mois de prélèvement, d'avril à décembre.

A-Projections of the physico-chemical variables of water samples collected on the plane of the first two axes of the PCA (axis 1 horizontal and axis 2 vertical). B-Projections of the three groups of stations, according to the measured physico-chemical variables, on the plane of the first two factorial axes of the PCA (axis 1 horizontal and axis 2 vertical). Numbers 4 to 9 indicate the months of the sampling, from April to December. 
groupes sont caractérisés par une pollution organique d'origine multiple, fondée sur des substances organiques biodégradables, et par une augmentation quelque peu appréciable des teneurs d'orthophosphates dans le cours moyen et une partie du cours inférieur. Pour la station Mf7, la zone marécageuse semble réduire notablement les valeurs de nombreuses variables indicatrices de la pollution de l'écosystème (Tableau 1).

\subsection{Diversité et structure de la communauté diatomique}

La microflore collectée pendant l'étude comporte 237 taxons différents appartenant à 39 genres de diatomées répartis dans 25 familles. Beaucoup d'espèces et variétés identifiées sont cosmopolites, accompagnées des formes tropicales telles que Cymbella tumida (Brebisson) Van Heurck, Gomphonema parvulum var. lagenula (Kützing) Frenguelli et Nitzschia tropica Hustedt (KRAMMER et LANGE-BERTALOT, 1986-1991). Plusieurs organismes appartenant aux genres Chaetoceros, Cymatopleura, Denticula, Diadesmis, Tetracyclus, Eunotia, Navicula, Nitzschia, Fragilaria, Frustulia, Pinnularia et Surirella n'ont pas pu être identifiés au niveau de l'espèce. Les recherches complémentaires devant conduire à leur identification se poursuivent. Les familles les plus représentées en ce qui a trait au nombre d'espèces sont les Naviculaceae $(14,71 \%)$, les Bacillariaceae $(13,87 \%)$, les Fragilariaceae $(10,92 \%)$, les Gomphonemataceae $(8,82 \%)$, les Pinnulariaceae $(8,40 \%)$, les Eunotiaceae $(7,14 \%)$, les Achnanthaceae $(7,14 \%)$, et les Cymbellaceae $(5,88 \%)$. Les autres familles présentent chacune une abondance relative inférieure à $4 \%$.

La variation spatiale de la richesse spécifique (RS), de l'indice de diversité de Shannon et Weaver ( $\left.\mathrm{H}^{\prime}\right)$, de l'équitabilité (E), et la proportion d'espèces polluo-tolérantes (\%VTP) est présentée dans le tableau 2. La richesse spécifique des trois premières stations (Mf1 à Mf3) comportant de 28 à 31 espèces ou taxons infra-spécifiques, est faible comparée à celle des stations du cours inférieur, où se développent un plus grand nombre d'espèces. L'indice H' fluctue entre 2,22 (Mf5) et 3,92 (Mf7) et révèle une diversité élevée dans les stations Mf2 à Mf4, et Mf7. L'équitabilité E présente une évolution similaire à celle de l'indice $\mathrm{H}$ ' et indique par ailleurs que ces mêmes stations Mf2 à Mf4 et surtout Mf7 renferment des espèces qui sont mieux réparties en matière d'effectifs et qui dominent le peuplement diatomique. Le \%VTP s'accroît sensiblement de la source à l'aval du cours d'eau. Les stations Mf2 à Mf6 montrent de fortes valeurs du \%VTP (de 61,42 à 77,50), ce qui est à rapprocher de l'apport important en substances organiques qui favorisent le développement des espèces saprophiles et indicatrices de l'enrichissement du milieu en nutriments. On observe que toutes les variables décrivant la richesse, la diversité et l'équitabilité des espèces augmentent le long du dernier tronçon (Mf6 à Mf7) qui correspond à la zone marécageuse. À

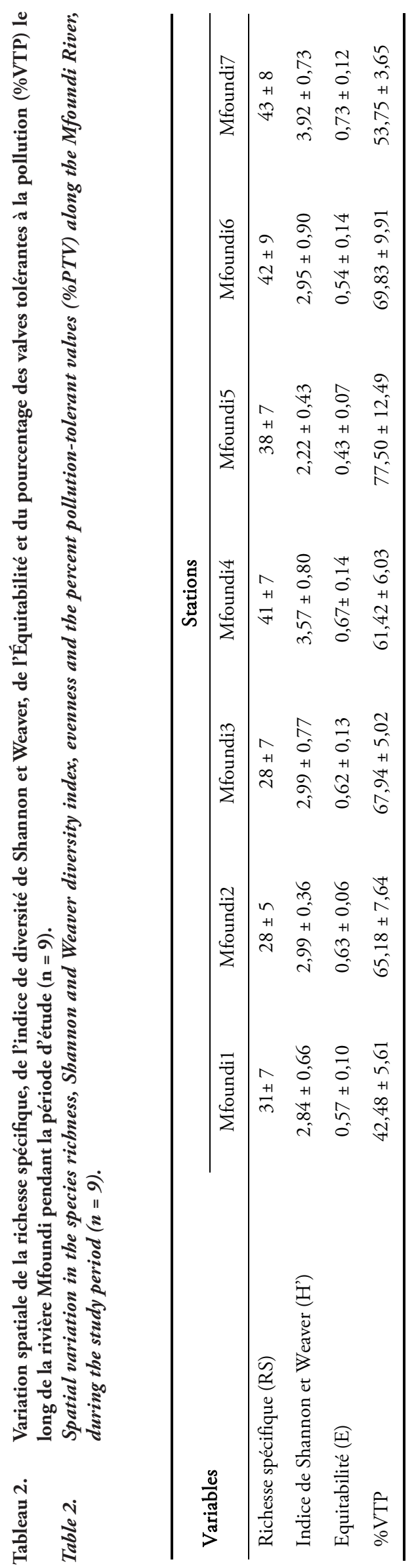


l'opposé, le pourcentage d'espèces polluo-tolérantes diminue. La variation de ces indicateurs traduit l'effet d'assainissement qu'exerce le marécage sur le flux polluant, et donc sur les communautés algales.

L'analyse en composantes principales (ACP), effectuée à partir de 44 espèces et variétés de diatomées (ayant une abondance relative cumulée supérieure ou égale à $2 \%$ ), a permis de visualiser les grands traits de la distribution spatiotemporelle de ces taxons (Figure 3A). Les deux premiers axes factoriels de l'ACP représentent 26,31\% de la variabilité expliquée et séparent les stations en trois groupes caractérisés par des assemblages différents de diatomées (Figure 3B) : Le groupe 1 correspond à la station Mf1 caractérisée par la prédominance à toutes les saisons de Gomphonema gracile Ehrenberg (GGRA), Gomphonema parvulum Kützing var. parvulum f. parvulum (GPAPP), Gomphonema parvulum var. exilissimum Grunow (GPAE) et Gomphonema parvulum var. parvulius Lange-Bertalot et Reichardt (GPAP). Ces espèces et variétés sont accompagnées de Navicula radiosa Kützing (NRAD), Navicula heimansioïdes Lange-Bertalot (NHES) et Gomphonema parvulum var. parvulum $f$. saprophilum LangeBertalot et Reichardt (GPAPS) qui se développent de préférence pendant la saison des pluies. Le groupe 2 réunit la gamme des stations Mf2 à Mf6. Ces stations présentent un peuplement diatomique dominé en toute saison par l'association Nitzschia palea (Kützing) W. Smith (NIPA), Gomphonema parvulum var. lagenula (Kützing) Frenguelli (GPAL), Navicula geoppertiana (Bleisch) Grunow (NAGE), Navicula mutica Kützing (NMUT) et Pinnularia subcapitata Gregory (PSUB). D’autres diatomées présentent un développement plus localisé, limité à une ou deux stations, à certaines périodes de l'année. C'est le cas de Fragilaria biceps (Kützing) Lange-Bertalot, Fragilaria ulna var. acus (Kutzing) Lange-Bertalot, Fragilaria ulna (Nitzsch) Lange-Bertalot var. ulna et Navicula menisculus Schumann à la station Mf2 aux mois de mai et octobre. La station Mf3 est favorable à la multiplication pendant la grande saison des pluies notamment en septembre et octobre des "petites Navicula spp " : Navicula seminuloides Hustedt (NSES), Navicula dictadetenta Hustedt (NDICT), Navicula minuscula Grunow (NMIN) et Navicula atomus var. permitis (Hustedt) LangeBertalot (NATP). Les stations Mf2 et Mf4 quant à elles sont caractérisées par le développement de Amphora montana Krasske (AMMO), Nitzschia frustulum (Kützing) Grunow (NIFRU) et quelques taxons planctoniques tels que Aulacoseira granulata (Ehrenberg) Simonsen (AUGR) et Cyclotella kuetzingiana Twaites (CYKU). Fragilaria construens $f$. venter (Ehrenberg) Hustedt (FRCOV) se développe de préférence au mois de novembre à la station Mf6. Le groupe 3 isole la station Mf7, caractérisée par la dominance de Diadesmis contenta (Grunow ex. V. Heurck) Mann (DICO), Achnanthes minutissima Kutzing (ACMI), Navicula cryptocephala Kützing (NCRY), Navicula pseudoventralis Hustedt (NPSVE) et certains taxons enregistrés dans les sites amont mais avec de faibles effectifs ( $N$. frustulum, $N$. geoppertiana, G. parvulum var. parvulum f. parvulum).

Considérant l'ensemble des prélèvements, la figure 4 résume la fréquence de distribution et la substitution des principales espèces et variétés de diatomées le long du gradient de pollution. À la station Mf1, l'association $G$. parvulum var. parvulum f. parvulum (29,6 \%), G. parvulum var. exilissimum $(24,18 \%), G$. parvulum var. parvulius $(10,74 \%)$ et $G$. gracile $(6,36 \%)$ prédomine. L'abondance relative de ces taxons baisse dans le cours inférieur. P. subcapitata se développe davantage dans les stations Mf2 (8,88 \%) et Mf3 (7,31\%). N. mutica et $N$. geoppertiana présentent des abondances élevées à la station Mf3. G. parvulum var. lagenula est bien représentée dans le secteur aval du Mfoundi, et est moins abondante à la station Mf1. N. palea se développe de façon optimale dans les cours moyen et inférieur de la rivière, son abondance relative atteignant $66,41 \%$ et $52,80 \%$ dans les stations Mf5 et Mf6 respectivement. Cette espèce est par contre peu fréquente dans la station Mf1 (1,59\%). Le peuplement diatomique de la station Mf7 est dominé par D. contenta (13,93\%), les taxons enregistrés dans les stations amont n'y présentant que de faibles abondances.

\subsection{Florule diatomique et qualité physico-chimique des eaux}

Le tableau 3 présente le coefficient de corrélation de rang (rs) de Spearman (pour $\mathrm{p}<0,05$ et $\mathrm{p}<0,01$ ) calculé entre certaines variables physico-chimiques de l'eau et l'abondance relative des onze taxons de diatomées représentant au minimum $5 \%$ de la récolte. Ces résultats montrent que les assemblages diatomiques du Mfoundi présentent des degrés différenciés de sensibilité aux conditions environnementales de leurs habitats. Ainsi, l'abondance de $N$. palea (NIPA) présente des corrélations assez fortes et positives ( $r s \geq 0,71$ ) avec huit des facteurs abiotiques étudiés à savoir, la conductivité électrique, l'alcalinité, la dureté totale, la dureté calcique et magnésienne, l'azote ammoniacal, les orthophosphates et l'oxydabilité. Cette espèce semble mieux se développer lorsque ces huit variables présentent des valeurs élevées. Elle serait donc indicatrice des eaux à la fois chargées de matières organiques et très minéralisées. La même espèce montre une corrélation négative avec la température, les matières en suspension, la couleur de l'eau et la turbidité. Ces variables sont souvent positivement corrélées à l'abondance de certains taxons.

Plusieurs variétés de G. parvulum, notamment G. parvulum var. parvulum $f$. parvulum, $G$. parvulum var. parvulius et $G$. parvulum var. exilissimum, ont montré en général, à l'opposé, des corrélations négatives avec les indicateurs de pollution organique. Ces taxons apparaissent plus sensibles à la détérioration de la qualité de l'eau par les matières organiques et les alcalino-terreux. Ils sont par ailleurs positivement corrélés 

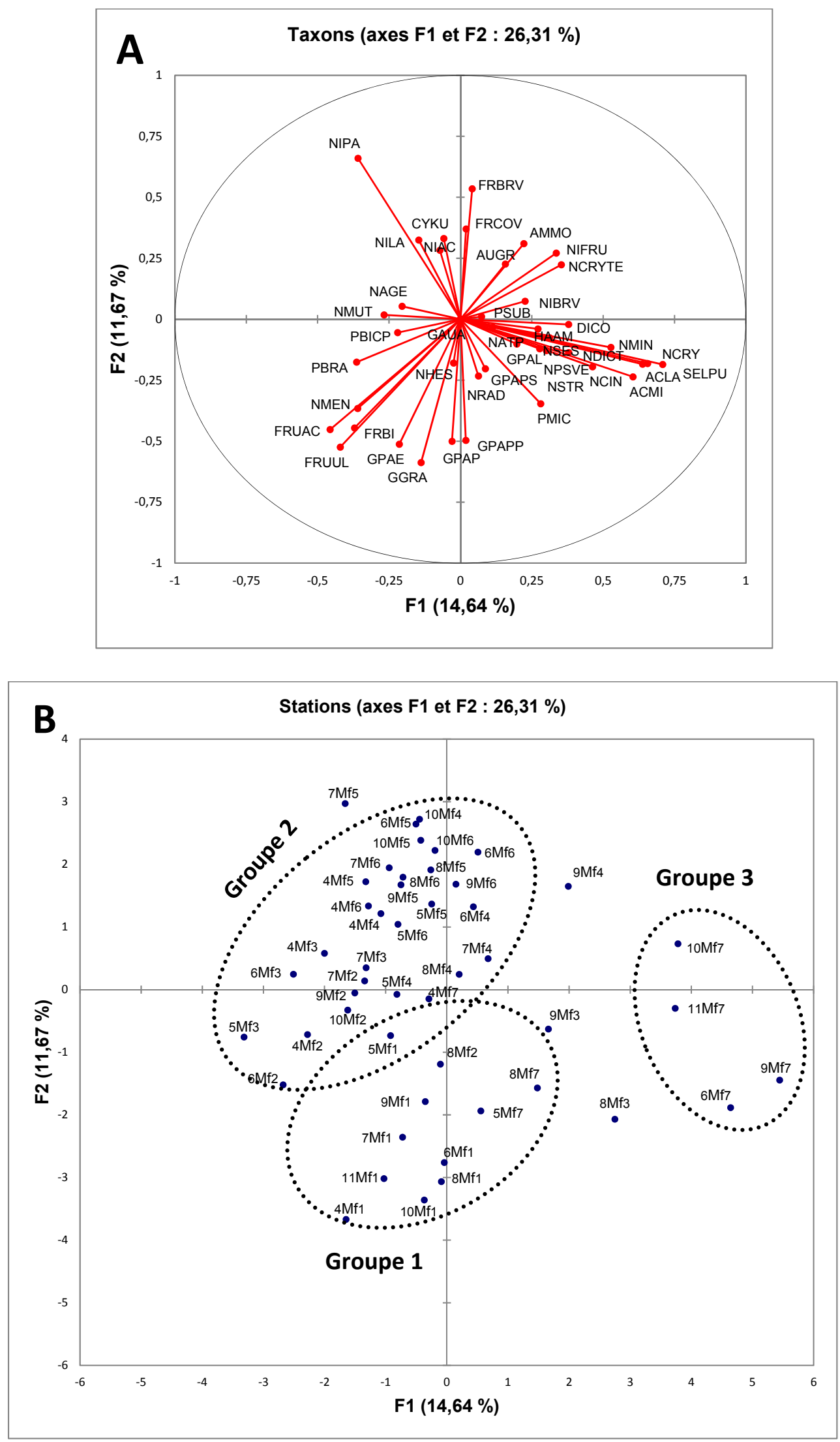

Figure 3. A- Projections des espèces et variétés de diatomées ( $\geq 2 \%$ d'abondance relative cumulée) collectées pendant l'étude dans les sept stations le long de la rivière Mfoundi, sur le plan de deux premiers axes factoriels de l'ACP (axe 1 horizontal et axe 2 vertical). B- Projections des stations, décrites par leurs communautés diatomiques, sur le plan de deux premiers axes factoriels de l'ACP (axe 1 horizontal et axe 2 vertical). Les nombres 4 à 9 précédant la station représentent le mois de prélèvement, d'avril à décembre.

A-Projections of diatom taxa ( $\geq 2 \%$ of the cumulative relative abundance for the study period) on the plane of the first two factorial axes of the PCA (axis 1 horizontal and axis 2 vertical). See names of taxa in the text. B-Projections of the stations, described by their diatom assemblages, on the plane of the first two factorial axes of the PCA (axis 1 horizontal and axis 2 vertical). Numbers 4 to 9 indicate the months of the sampling, from April to December. 


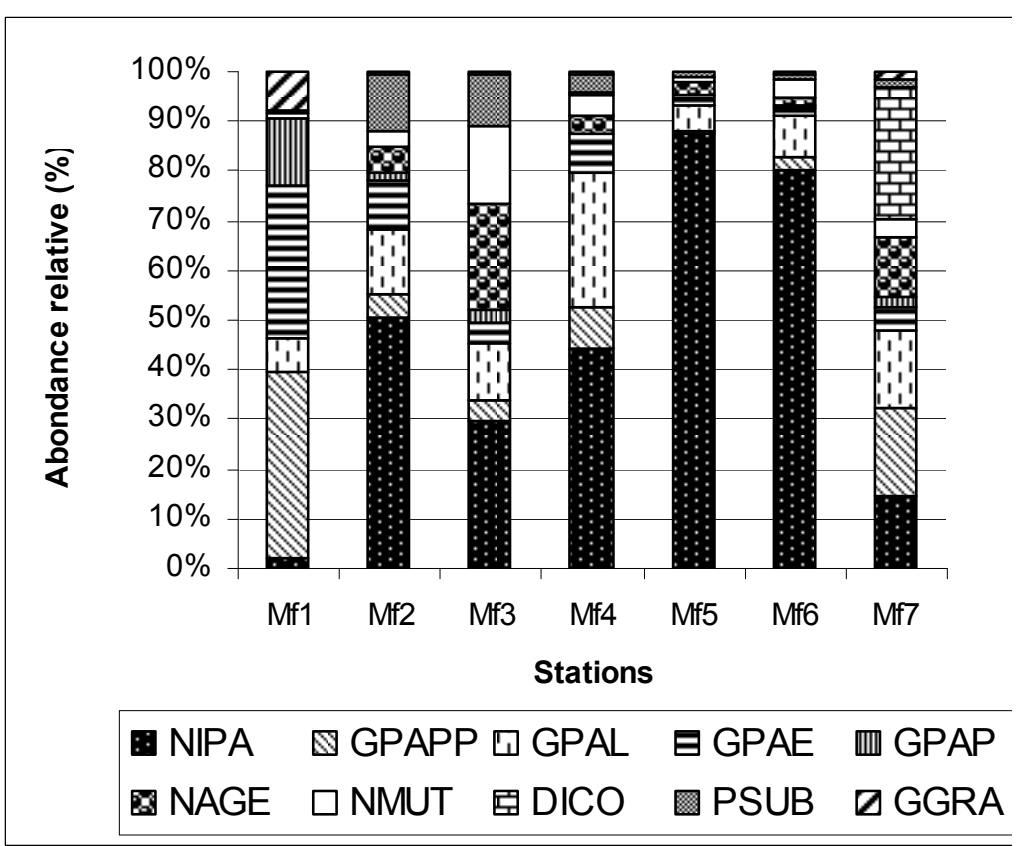

Figure 4. Variation de l'abondance relative moyenne (\%) des principales espèces et variétés de diatomées dans les sept stations le long de la rivière Mfoundi (n = 9). NIPA : Nitzschia palea; GPAPP : Gomphonema parvulum varparvulum f. parvulum; GPAL: Gomphonema parvulum var. lagenula; GPAE : Gomphonema parvulum var. exilissimum; GPAP : Gomphonema parvulum var. parvulum; NAGE : Navicula geoppertiana; NMUT : Navicula mutica; DICO : Diadesmis contenta; PSUB : Pinnularia subcapitata; GGRA : Gomphonema gracile. Variation of the mean relative abundance (\%) of the main diatom species at the sampling stations along the Mfoundi River $(n=9)$.

avec les matières en suspension, la couleur de l'eau et la turbidité. En plus de ces trois variables, le développement de $G$. parvulum var exilissimum est favorisé par une augmentation de $\mathrm{pH}(\mathrm{rs}=0,80)$ et par les ions magnésium $(\mathrm{rs}=0,74)$; on relève que la température constitue un facteur limitant pour cette variété et pour $G$. parvulum var parvulius. L'abondance de $G$. parvulum var lagenula est quant à elle corrélée positivement à la couleur de l'eau ( $\mathrm{rs}=0,81)$, la dureté magnésienne $(\mathrm{rs}=0,69)$ et l'oxydabilité ( $r s=0,67$ ). Huit facteurs abiotiques se révèlent déterminants dans la distribution de G. gracile. Ce taxon présente des corrélations positives avec les matières en suspension, la couleur de l'eau, la dureté totale et magnésienne, alors que la conductivité, la dureté calcique, les ions orthophosphates et l'azote ammoniacal lui sont corrélés négativement.

La dureté totale, les ions orthophosphates et les matières en suspension sont favorables au développement de P. subcapitata. Un pH élevé, l'alcalinité, la turbidité et l'oxydabilité exercent au contraire une action limitante sur cette espèce. $N$. geoppertiana montre une corrélation positive très forte et significative (à $\mathrm{p}<0,01$ ) avec l'oxydabilité, et un besoin important d'ions orthophosphates, d'ammonium et de calcium pour son développement. L'abondance de $N$. mutica est associée surtout à la dureté magnésienne, à l'oxydabilité et, dans une moindre mesure, à la température et aux ions ammonium. Le développement de $D$. contenta est en général inhibé par la plupart des paramètres indicateurs de pollution organique. La variation d'abondance de N. cryptocephala le long du Mfoundi ne semble pas dépendre de la plupart des caractéristiques de l'eau prises en compte au tableau 3. Cependant, cette espèce montre des corrélations positives avec l'alcalinité ( $\mathrm{rs}=0,76)$ et l'oxydabilité ( $r s=0,86)$, et une corrélation négative avec la conductivité ( $r s=-0,68)$.

\section{DISCUSSION}

L'étude du peuplement diatomique de la rivière Mfoundi en relation avec les variables physico-chimiques de l'eau a permis une première analyse de l'écologie de l'épilithon et de la qualité de l'eau. La forte turbidité des eaux dans le secteur amont du 


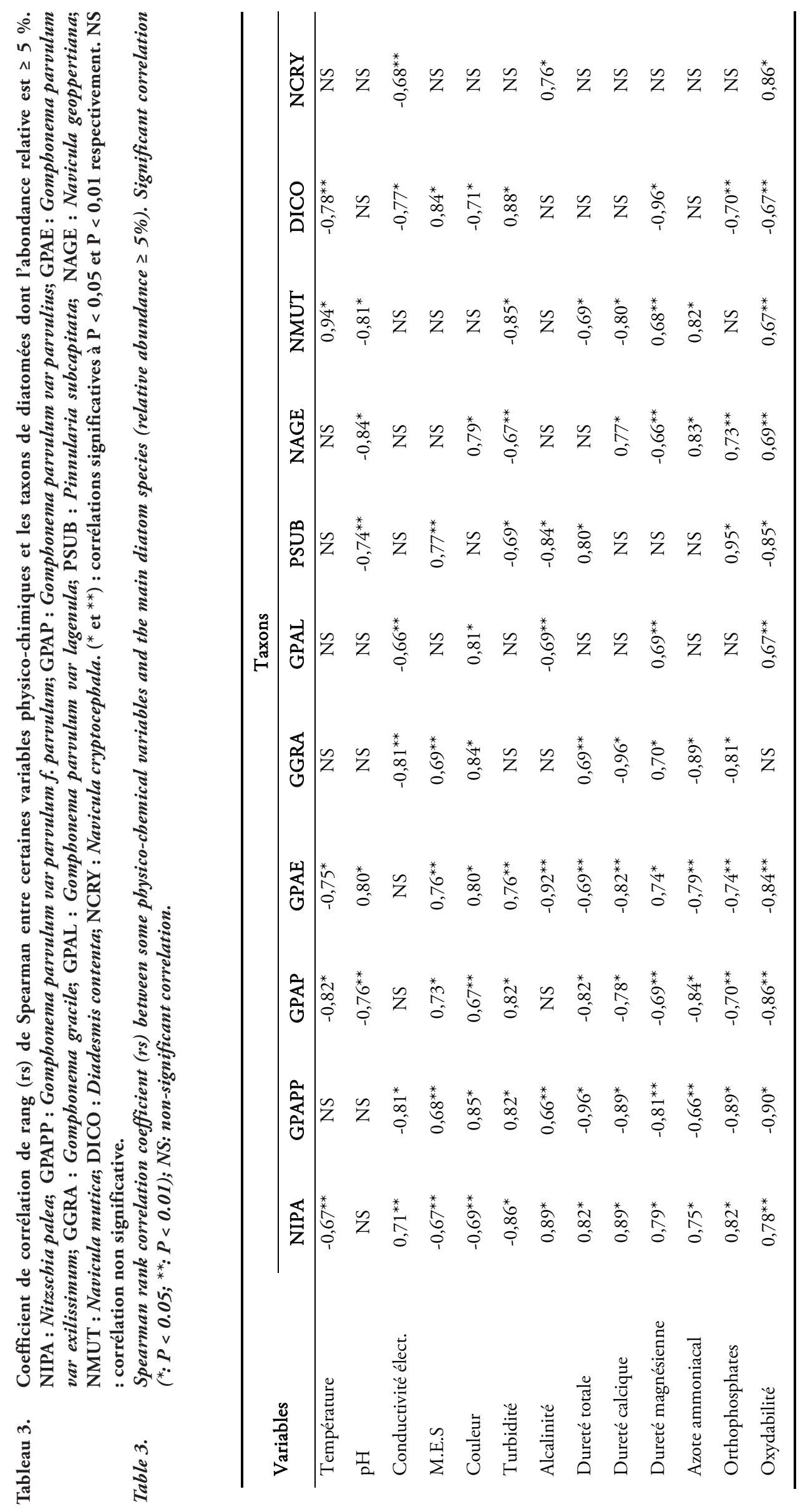


cours d'eau est à attribuer à l'érosion du sol des espaces cultivés (TCHOTSOUA, 1993), d'une part, et à l'apport des eaux usées domestiques, d'autre part.

Dans les cours moyen et inférieur, aux rejets d'eaux usées domestiques s'ajoutent les effluents d'industries alimentaires non traités. Ces rejets sont à l'origine de la pollution organique matérialisée par les valeurs élevées de l'oxydabilité et de la $\mathrm{DBO}_{5}$, les fortes teneurs de l'azote ammoniacal, et les teneurs très moyennes en orthophosphates. Ces dernières dans l'ensemble ne sont guère bien élevées. Mais l'on sait qu'en région équatoriale, l'énergie solaire étant permanente, de faibles doses de phosphore sont suffisantes pour assurer un bon développement des algues et des macrophytes (OCDE, 1982). La dégradation des composés organiques des eaux résiduaires entraine les faibles taux de saturation en oxygène dissous qui ont été enregistrés. Ces derniers résultats corroborent les observations de FOTO MENBOHAN (1997) tout en mettant en évidence une accentuation de la dégradation de la qualité de ces eaux depuis cette précédente étude. La légère amélioration observée à la station Mf7, après la traversée de la zone marécageuse, est sans doute le résultat des processus d'autoépuration dont les marécages sont le siège (FAWZI et al., 2001; GOMEZ, 1998; PULLIN et HAMMER, 1991), et qui ont un retentissement sur la diversité des espèces évoluant dans ces milieux.

Les résultats obtenus indiquent une grande variabilité spatiale de la diversité des espèces de diatomées qui peuplent les différentes stations d'étude. Ceci souligne la variabilité des conditions de milieu qui prévalent le long du cours d'eau, les taxa étant surtout distribués suivant le gradient de pollution organique. La faible diversité spécifique relevée à la station Mf1 serait due à plusieurs facteurs agissant en synergie : (1) les faibles teneurs en nutriments et en matières organiques à cette station, (2) la forte vitesse d'écoulement de l'eau qui ralentit la colonisation des substrats par les diatomées, augmentant ainsi la dérive de certains taxons (BIGGS et CLOSE, 1989; CLAUSEN et BIGGS, 1997; LELAND et PORTER, 2000), et (3) le broutage qui serait exercé par les larves de Trichoptères se développant sur les substrats. Plusieurs auteurs ont montré que les indicateurs de la diversité spécifique ne permettent pas toujours, à eux seuls, d'estimer l'impact des pollutions sur l'état de santé des cours d'eau (BELLINGER et al., 2006; GOMÀ et al., 2005; LOBO et KOBAYASI, 1990; LOBO et al., 1995). Ceux-ci doivent donc être associés à la composition spécifique des organismes étudiés et à une bonne connaissance de leur autoécologie (ARCHIBALD, 1972; COX, 1988).

Les communautés de diatomées rencontrées dans le Mfoundi ont des sensibilités différentes vis-à-vis des conditions du milieu et développent de ce fait des types morphologiques variés. Ainsi, certaines variétés de $G$. parvulum et de $G$. gracile se multiplient abondamment à la station Mf1 qui est la moins perturbée. Ces morphotypes de G. parvulum ont été positivement corrélés aux matières en suspension, à la turbidité et la couleur de l'eau. L'abondance de ces morphotypes de $G$. parvulum a été rapportée par SALOMONI et al. (2006) dans le cours supérieur de la rivière Gravataï au Brésil, et par NDIRITU et al. (2003) dans les eaux turbides de la rivière Nairobi au Kenya. Cette espèce dont les densités sont d'après KOBAYASI et MAYAMA (1989) et LOBO et al. (2004) généralement élevées dans les eaux chargées de matières organiques, et qui s'accommode donc des pollutions organiques (LANGEBERTALOT, 1979; VAN DAM et al., 1994), semble bien caractériser les eaux turbides et à faible minéralisation du cours supérieur du Mfoundi. Quant à l'espèce G. gracile, connue pour sa préférence pour les eaux oligotrophes, neutres à légèrement alcalines (SCHOEMAN, 1973), elle apparaît dans ce travail comme une espèce tolérant ce type de pollution.

Les espèces $N$. palea, N. mutica, N. geoppertiana, P. subcapitata et $G$. parvulum var. lagenula ont été fréquemment dénombrées dans les stations Mf2 à Mf6. Ces espèces sont considérées comme étant $\alpha$-méso/polysaprobes, termes qui décrivent leur préférence pour les milieux riches en matières organiques en décomposition. Selon LELAND et PORTER (2000) et VAN DAM et al. (1994), ces espèces prolifèrent dans les sections eutrophes voire hypereutrophes des hydrosystèmes, comme il en est dans les stations Mf2 à Mf6 du Mfoundi. Ceci pourrait permettre de comprendre pourquoi les abondances de $N$. palea sont positivement corrélées à la conductivité, aux ions ammonium, aux orthophosphates et à l'oxydabilité, à l'alcalinité et à la dureté de l'eau. Ces résultats corroborent les observations faites par BLINN et al. (2004) et MORIN (2006) dans diverses hydro-écorégions d'Australie et de France, respectivement.

D'une manière générale, les apports constants de nutriments et de substances organiques, par le biais des rejets d'eaux usées domestiques et d'effluents industriels, pourraient expliquer les fortes proportions d'espèces tolérantes à la pollution organique (\%PTV) qui ont été enregistrées dans cette étude. Le \%PTV est généralement utilisé pour estimer l'influence de la pollution organique sur l'indication de l'état trophique de la station de prélèvement. Il permet d'identifier les stations où la pollution organique est le principal facteur qui influence la composition des communautés diatomiques. Ce paramètre est conjointement utilisé avec les valeurs de l'indice diatomique trophique pour indiquer sa fiabilité comme métrique de l'eutrophisation de la station (KELLY et WHITTON, 1995; KELLY, 1998). Des résultats similaires ont été obtenus par KELLY (1998) en Grande-Bretagne et BEYENE et al. (2009) en Éthiopie. Ces auteurs ont noté un développement important des espèces polluo-résistantes dans les sites localisés en aval des stations d'épuration des égouts et dans les tronçons des cours d'eau urbains très polluées par les rejets domestiques et industriels. L'abondance de D. contenta et de $N$. cryptocephala, 
espèces couramment rencontrées dans les sections oligotrophes à eutrophes de divers hydrosystèmes (SOININEN, 2002; VAN DAM et al., 1994), est en faveur de l'amélioration de la qualité de l'eau de la station Mf7 déjà soulignée par les descripteurs physico-chimiques.

La variabilité saisonnière des diatomées dans le Mfoundi n'a pas été très marquée; la distribution de ces microorganismes étant plus conditionnée par les changements de la qualité de l'eau le long de la rivière. Cependant, le développement à la station Mf3 des formes prostrées (Navicula spp : N. seminuloides, $N$. dictadetenta, $N$. minuscula et $N$. atomus var. permitis), très adhérentes aux substrats pendant la grande saison des pluies, serait attribué aux brusques variations de débits et au brassage des eaux (EULIN, 1997).

\section{CONCLUSION}

L'étude sur le peuplement diatomique du Mfoundi, cours d'eau urbain, révèle la grande richesse des espèces qui constituent ce groupe d'organismes, en réponse aux conditions de milieu qui prévalent le long du cours d'eau.

\section{REMERCIEMENTS}

Cette recherche a été financée partiellement par la Fondation Internationale pour la Science (FIS), Suède, par l'intermédiaire d'une bourse de recherche accordée à Daniel EBANG MENYE (Réf. : A/4606-1). Les auteurs remercient également les experts anonymes dont les multiples critiques et suggestions ont permis d'améliorer la qualité du manuscrit.

\section{RÉFÉRENCES BIBLIOGRAPHIQUES}

AFNOR (2003). Qualité de l'eau. Guide pour l'échantillonnage en routine et le pré-traitement des diatomées benthiques des rivières. Normes NF EN 13946, 16 p.

AGENDIA L.P., T. FONKOU, D.J. SONWA, R. MEFENYA, Y.M. KENGNE et A.J.M. ZAMBO (2000). Collecte, épuration et évacuation des eaux usées dans les lotissements Sic-MAETUR de Yaoundé. Rev. Geogr. Cam., 2, 173-192.

APHA-AWWA (1985). Standard methods for the examination of water and wastewater. APHA-AWWA-WPCF, Pennsylvanie, Washington, DC, États-Unis, 1150 p.
ARCHIBALD R.E.M. (1972). Diversity in some South African diatom associations and its relation to water quality. Water Res., 6, 1229-1238.

BACHELIER G. (1959). Étude pédologique des sols de Yaoundé. Contribution à l'étude de la pédogenèse des sols ferralitiques. Agron. Trop., 19, 279-305.

BELLINGER B.J., C. COCQUYT et C.M. O'REILLY (2006). Benthic diatoms as indicators of eutrophication in tropical streams. Hydrobiologia, 573, 75-87.

BEYENE A., T. ADDIS, D. KIFLE, W. LEGESSE, H. KLOOS et L. TRIEST (2009). Comparative study of diatoms and macroinvertebrates as indicators of severe water pollution: Case study of the Kabena and Akaki rivers in Addis Ababa, Ethiopia. Ecol. Indic., 9, 381-392.

BIGGS B.J.F. et M.E. CLOSE (1989). Periphyton biomass dynamics in gravel bed rivers: the relative effects of flow and nutrients. Freshwater Biol., 22, 209-231.

BLINN D.W et P.C.E. BAILEY (2001). Land-use influence on stream water quality and diatom communities in Victoria, Australia: a response to secondary salinization. Hydrobiologia, 466, 231-244.

BLINN D.W., S.A HALSE., A.M. PINDER, R.J. SHIEL et J.M. McRAE (2004). Diatom and micro-invertebrate communities and environmental determinants in the western Australian wheatbelt: a response to salinization. Hydrobiologia, 528, 229-248.

BOURRELLY P. (1968). Les algues d'eau douce: les algues jaunes et brunes. Boubée et Cie, Paris, France, 438 p.

CAMARGO J.A. (1994). The importance of biological monitoring for the ecological risk assessment of freshwater pollution: a case study. Environ. Int., 20, 229-238.

CARTER J.R et P. DENNY (1982-1992). Freshwater algae of Sierra Leone, IV. Bacillariophyceae. 1: Diatoms from the River Jong (Taia) at Njala, 331p; 2: diatoms from the coastal region of the Southern Province, 46p; 3: diatoms from the Lake Sonfon region and from Lake Popei, 52 p. Nova Hedwigia, Stuttgart, Germany.

CLAUSEN B et B.J.F. BIGGS (1997). Relationships between benthic biota and hydrological indices in New Zealand streams. Freshwater. Biol., 38, 327-342.

COMPÈRE P. (1975). Algues de la région du lac Tchad IVDiatomophycées. Cah. ORSTOM Ser. Hydrobiol., IX, 203290. 
COX E.J. (1988). Microdistributional patterns of freshwater diatoms in relation to their use as bioindicator. Dans : Proceedings of the $10^{t h}$ International Diatom Symposium (ed. H. Simola), pp. 521-528. Koeltz, Koenigstein, Joensuu, Finland.

DAJOZ R. (2000). Précis d'écologie. Dunod, Paris, France, $615 \mathrm{p}$.

DE LA REY P.A, J.C. TAYLOR., A. LAAS, L. VAN RENBURG et A. VOSLOO (2004). Determining the possible application value of diatoms as indicators of general water quality - A comparison with SASS 5. Water $S A, 30,325-332$.

DÉCRET 165/PM (2001). Modalités de protection des eaux de surface et des eaux souterraines contre les pollutions en République du Cameroun. 9 p.

EULIN A. (1997). Les communautés de diatomées épilithiques de la Garonne. Répartition naturelle et étude expérimentale in situ de la dynamique de colonisation sur substrat artificiel. Thèse de Doctorat, Univ. Paul Sabatier, Toulouse, France, $250 \mathrm{p}$.

FAWZI B., M. CHLAIDA, S. OUBRAIM, M. LOUDIKI, B. SABOUR et A. BOUZIDI (2001). Application de certains indices diatomiques à un cours d'eau marocain : Oued Hassar. Rev. Sci. Eau, 14, 73-89.

FOTO MENBOHAN S. (1997). La pollution de deux cours d'eau urbains au Cameroun : l'Abiergué et le Mfoundi. Aspect physico-chimique. Cam. J. Bioch. Sci., 7, 1-13.

GASSE F. (1986). East African diatoms: Taxonomy, Ecological distribution, bibliotheca Diatomologica. J. Cramer, Berlin, $201 \mathrm{p}$.

GOMEZ N. (1998). Use of epipelic diatoms for evaluation of water quality in the Matanza-Riachuelo (Argentina), a pampean plain river. Water Res., 32, 2029-2034.

GOMÀ J., F. RIMET, J. CAMBRA, L. HOFFMANN et L. ECTOR (2005). Diatom communities and water quality assessment in mountain rivers the upper Sègre basin (La Cerdanya, Pyrénées). Hydrobiologia, 551, 209-225.

HARDING W.R., C.G.M. ARCHIBALD et J.C. TAYLOR (2005). The relevance of diatoms for water quality assessment in South Africa: A position paper. Water $S A$, $31,41-46$.

JÜTTNER I., H. ROTHFRITZ et S.J. ORMEROD (1996). Diatoms as indicators of river quality in the Nepalese
Middle Hills with consideration of the effects of habitatspecific sampling. Freshwater Biol., 36, 475-486.

KELLY M.G. (1998). Use of the trophic diatom index to monitor eutrophication in rivers. Water Res., 32, 236-242.

KELLY M.G. et B.A. WHITTON (1995). The trophic diatom index: a new index for monitoring eutrophication in rivers. J. Appl. Phycol., 7, 433-444.

KOBAYASI H. et S. MAYAMA (1989). Evaluation of river water quality by diatoms. Korean J. Phycol., 4, 121-133.

KRAMMER K. et H. LANGE-BERTALOT (1986-1991). Süßwasserflora von Mitteleuropa. Bacillariophyceae, 2/1: Naviculaceae, 876 p.; 2/2: Nitzschiaceae, Epithemiaceae, Surirellaceae, 596 p.; 2/3: Centrales, Fragilariaceae, Eunotiaceae, 576 p.; 2/4: Achnanthaceae, 437 p. G. Fischer, Stuttgart, Germany.

LANGE-BERTALOT H. (1979). Pollution tolerance of diatoms as a criterion for water quality estimation. Nova Hedwigia Beihefte., 64, 285-304.

LANGE-BERTALOT H. et D. METZELTIN (1996). 800 Taxa representative of three ecologically distinct lake types: carbonate buffered-oligodystrophic-weakly buffered soft water. Iconographia Diatomologica: annotated diatom micrographs (Vol.2), Berlin, Germany, 390 p.

LELAND H.V. et S.T. PORTER (2000). Distribution of benthic algae in the Illinois River basin in relation to geology and land use. Freshwater Biol., 44, 279-301.

LE PIMPEC P., A. LIÉNARD, R. BONNARD, M. LAFONT, B. CAZIN, P. BOSSARD, B. HUBERT et M. BRAY (2002). Guide de l'agent préleveur chargé de la police des milieux aquatiques. Édition Maurice Merlin, France, 159 p.

LOBO E.A. et H. KOBAYASI (1990). Shannon's diversity index applied to some freshwater diatom assemblages in the Sakawa River system (Kanagawa, Japan) and its use as an indicator of water quality. Japan. J. Phycol., 381, 229-243.

LOBO E.A., K. KATOH et Y. ARUGA (1995). Response of epilithic diatom assemblages to water pollution in rivers in the Tokyo Metropolitan area. Japan Freshwater Biol., 34, 191-204.

LOBO E.A., D. BES, L. TUDESQUE et L. ECTOR (2004). Water quality assessment of the Pardinho river, RS, Brazil, using epilithic diatom assemblages and faecal coliforms as biological indicators. Vie Milieu, 54, 105-114. 
MORIN S. (2006). Bioindication in situ des effets des pollutions métalliques sur les communautés de diatomées benthiques: approches in situ et expérimentales. Thèse de Doctorat, Univ. Bordeaux 1, France, 302 p.

NDIRITU G.G., N.N. GICHUKI, P. KAUR et L. TRIEST (2003). Characterization of environmental gradients using physico-chemical measurements and diatom densities in Nairobi River, Kenya. Aquat. Ecosyst. Health Manag., 6, 343-354.

NGUÉTSOP V.F. (1997). Évolution des environnements de l'Ouest-Cameroun depuis 6000 ans, d'après l'étude des diatomées actuelles et fossiles dans le lac Ossa. Implications paléoclimatiques. Thèse de Doctorat, Muséum National d'Histoire Naturelle, Paris, France, 277 p.

OCDE (1982). Eutrophication of waters: monitoring, assessment and control. Final report - Environmental Directorate, OCDE, Paris, France, 154 p.

OLIVRY J.C. (1986). Fleuves et rivières du Cameroun. Collection Monographies Hydrologiques ORSTOM No 9. MESRES-ORSTOM, Paris, France, 733 p.

PULLIN B.P. et B. HAMMER (1991) Aquatic plants improve wastewater treatment. Water Environ. Technol., 3, 36-40.

RODIER J. (1996). L'analyse de l'eau : eaux naturelles, eaux résiduaires et eau de mer. Chimie, physico-chimie, interprétation des résultats. $8 \mathrm{e}$ édition, Dunod, Paris, France, $1384 \mathrm{p}$.

ROTT E., H.C. DUTHIE et E. PIPP (1998). Monitoring organic pollution and eutrophication in the Grand River, Ontario, by means of diatoms. Can. J. Fish. Aquat. Sci., 55, 1443-1453.

ROUND F.E. (1991). Diatoms in river water-monitoring studies. J. Appl. Phycol., 3, 129-145.

SALOMONI S.E., O. ROCHA, V.L. CALLEGERO et E.A. LOBO (2006). Epilithic diatoms as indicators of water quality in the Gravatai River, Rio Grande do Sul, Brazil. Hydrobiologia, 559, 233-246.

SCHOEMAN F.R. (1973). A systematical and ecological study of the diatom flora of Lesotho with special reference to the water quality. V \& R Printers, Pretoria, South Africa, 365 p.

SHANNON C.E. et W. WEAVER (1949). The mathematical theory of communication, Univ. Illinois Press, Urbana, Illinois, USA, $117 \mathrm{p}$.
SOININEN J. (2002). Response of epilithic diatom communities to environmental gradients in some Finnish rivers. Int. Rev. Hydrobiol., 87, 11-24.

SUCHEL J.B. (1987). Les climats du Cameroun. Thèse de Doctorat d'État, Univ. Bordeaux III, France, 1186 p.

TANAWA E., H.B. DJEUDA TCHAPNGA, E. NGNIKAM, H. TCHAKOUNTIO, G. WOUATSA, H. BOTTA, J.M. DELEUIL, C. BERDIER et A.B. SOUHAM (2003). Gestion et valorisation des eaux usées dans les zones d'habitat planifié et leurs périphéries. Rapport final - Assainissement A08, Yaoundé, Cameroun, 167 p.

TCHOTSOUA M. (1993). Érosion accélérée et contraintes à l'aménagement du site de la ville de Yaoundé: une contribution à la gestion de l'environnement urbain en milieu tropical humide. Thèse de Doctorat $3^{\mathrm{e}}$ Cycle, Univ. Yaoundé 1, Cameroun, 286 p.

VAN DAM H., A. MERTENS et J. SINKELDAM (1994). A coded checklist and ecological indication values of freshwater diatoms from Netherlands. Neth. J. Aquat. Ecol., 28,117-133.

VICAT J.P. et P. BILONG (1998). Esquisse géologique du Cameroun. Dans : Géosciences au Cameroun, VICAT J.P. et BILONG P. (Éditeurs). Presse Univ. Yaoundé I, 454 p.

WINTER J.G et H.C. DUTHIE (1998). Effects of urbanization on water quality, periphyton and invertebrate communities in a Southern Ontario stream. Can. Water Res. J., 23,235-257. 